\title{
Boccaccio (comico) nel teatro (comico) di Machiavelli
}

\author{
Daria Perocco \\ Università Ca’ Foscari di Venezia \\ perocco@unive.it
}

\begin{abstract}
Molti critici hanno posto in parallelo Boccaccio e Machiavelli: cronologicamente, il primo testo trovato è una lettera di Girolamo Muzio del 1535. Oltre alla presenza del Decameron nella Mandragola, la cui influenza viene ulteriormente sottolineata, lo studio è orientato in funzione della Clizia, in cui lo stesso Machiavelli sembra allontanarsi e sfuggire dalle situazioni ironicamente boccaccesche che egli stesso aveva poco tempo prima creato per rifugiarsi in un linguaggio estremamente più piano e posato, quello che stava diventando predominante, dell'imitazione plautina e terenziana. A proposito viene anche riportato un significativo passo della traduzione dell'Andria. Vengono poi esaminati alcuni temi chiave: le donne, i vecchi e i giovani, la paura della maldicenza, le abilità sessuali dei personaggi, i giochi linguistici di attesa che implicano una teatralità dirompente, tutti letti nella ripresa più o meno letterale (ma sempre indicata) dai testi di Boccaccio. La conclusione è che Machiavelli, nel riprendere il Boccaccio comico, si diverte nel compiere un gioco equivoco con i termini usati: l'equivoco può sembrare della stessa natura, ma in un autore può addirittura diventare parodia del significato di quello che è nell'altro.
\end{abstract}

Parole chiave: Clizia, Mandragola, Machiavelli, Muzio, Donne in Machiavelli, Boccaccio comico.

\section{Abstract}

Many critics have placed Boccaccio and Machiavelli side by side. Chronologically, the first text we find is a letter written by Girolamo Muzio in 1535. Besides the presence of the Decameron in Mandragola, the influence is further underlined as we allow Clizia to guide the study. In this work Machiavelli seems to distance himself and flee from ironically Boccaccio-esque situations that he himself had created just slightly earlier in order to take refuge in extremely flat and composed language. This sober language which imitated Plautus and Terrence was becoming dominant at the time. In this line of investigation a significant passage from the translation of Andria is analyzed. Then some key themes are examined: women, old age and youth, fear of curses, the sexual skill of the characters, the linguistic waiting games involving unsettling theatrics, all of which are taken more or less literally (but always clearly) from the texts of Boccaccio. The conclusion is that in taking from Boccaccio's comedies, Machiavelli enjoys playing a game of ambiguity with the terms used. This ambiguity may seem to be of the same nature, but in it one author can even parody the meaning of the other author.

Key words: Clizia, Mandragola, Machiavelli, Muzio, Women in Machiavelli, comic Boccaccio. 
L'iterazione dell'aggettivo "comico» del titolo vuole, pur nella indubbia cacofonia della ripetizione, immediatamente rievocare la firma posta da Machiavelli nella lettera (post 21 ottobre 1525) a Francesco Guicciardini: «Niccolò Machiavelli istorico, comico e tragico»: nulla del «tragico» Machiavelli, da lui sparso nelle sue opere più diverse (anche se nessuna tragedia, nel senso tradizionale e aristotelico del termine egli scrisse), di quella "forma tragica» tanto, anch'essa, ripresa da Boccaccio dovrebbe qui comparire; mi propongo semplicemente di evidenziare qualche elemento recuperato, utilizzato e rigiocato per i «badalucchi» che il segretario fiorentino ha offerto ai suoi (reali) spettatori nelle sue (vere) commedie, nate e sviluppate per la scena.

Nel tempo trascorso da quando ho indicato il tema del mio intervento sono stati pubblicati due libri dedicati a Machiavelli che, in paragrafi riservati appositamente al testo della Mandragola, rammentano il tema della presenza di Boccaccio nel capolavoro teatrale del cinquecento, a conferma, se mai ce ne fosse bisogno, che la ricerca delle fonti e della genesi di un capolavoro letterario è argomento di studio che non si esaurisce e che continua ad attirare, facendo trovare sempre nuovi spunti e nuove prospettive di lettura. ${ }^{1}$

Il testo che, credo per primo, ha accostato, ponendoli in parallelo, lo «stilo» di Machiavelli e quello di Boccaccio è un brano che la problematica della cosiddetta «Questione della lingua nel Cinquecento» ha reso abbastanza famoso: si tratta della lettera di Gerolamo Muzio a Gabriele Cesano e Bartolomeo Cavalcanti che, già all'altezza cronologica del 1535, proponeva un chiaro rapporto tra i due autori (Boccaccio e Machiavelli) e li faceva contendere «l'un contro l'altro armati». Il testo fu divulgato e diffuso e, di conseguenza, divenne famoso una volta pubblicato nella raccolta dei testi linguistici scritti dal 1533 al 1574 col titolo di Battaglie in difesa dell'italica lingua (il merito della sua diffusione postuma va al figlio di Muzio, Giulio Cesare. Essi continuano, pur nella diversità dell'arco degli anni di scrittura, ad attestare la convinzione dell'autore che il volgare letterario in cui si deve scrivere è una lingua italiana (e non fiorentina), estremamente alta e colta, che il letterato acquista con lo studio dei suoi autori maggiori ("...la lingua degli scrittori [è] la più perfetta e [...] coloro che dagli scrittori la lingua apprendono, la più perfetta apprendano»). Ovvia quindi, di conseguenza, la denigrazione della prosa di Machiavelli e l'esaltazione di quella del Boccaccio, senza alcuna notazione che si premurasse di sottolineare come, in realtà, il primo fosse nutrito della «leggiadria» del secondo.

Vi dirò liberamente il vero (fate pur di me, dal giudicio ch'io fo di lui, quel giudicio vi piace, ché, se il dir del Boccaccio vi dispiace, non mi dispiace che il mio giudicio vi dispiaccia; e se lo stilo del Macchiavelli vi piace, non mi piace che la openion mia vi habbia a piacere): io non so trovar nelle parole di lui [scil. Machiavelli] cosa che comportabile mi paia in iscrittore che voglia con lode alcuna cosa scrivere. Se riguardo alla forma del dire, non so come dir si possa più

1. Sto alludendo a Luciano BotToni, La Messinscena del Rinascimento II. Il segreto del diavolo e "La Mandragola», Milano: Franco Angeli, 2006 e Giorgio IngLeSE, Per Machiavelli. L'arte dello stato, la cognizione delle storie, Roma: Carocci, 2006. 
bassamente. Se cerco de gli ornamenti non ne trovo niuno, anzi mi pare egli esser tutto secco e digiuno di ogni leggiadria. Poi nella lingua egli è tale che, oltra l'usar molte parole latine, là dove non men belle ne haverebbe havute delle volgari, et nella variatione et nella proprietà de' verbi egli è tutto cieco; usa male i nomi e peggio i pronomi; non sa ben collocare né articoli né adverbij, et in somma tanto fa delle osservationi della lingua quanto chi non ne sa niente. ${ }^{2}$

Credo che il povero Muzio avesse presenti soprattutto gli scritti cosiddetti politici del Segretario fiorentino, quelli nei quali «niuna pietà, niuna humanità, niuna religione vi si trova; ma che sono tutti pieni di ammaestramenti di crudeltà, di tirannia e di infidelità», ${ }^{3}$ frase che potremmo leggere, distorcendone totalmente il significato dalla lettura che il critico cinquecentesco avrebbe voluto si potesse ipotizzare nelle sue più recondite intenzioni: e quindi con una visione moderna e del tutto positiva, nel significato prettamente machiavelliano che appunto esorta a conoscere la via dell'Inferno per fuggirlo, significato che va ovviamente esteso anche alle commedie, in primis alla Mandragola.

Non sono stati pochi nel corso dei secoli, in compenso, gli studiosi che hanno posto in parallelo i due autori, notando la presenza delle situazioni comiche di derivazione decameroniana nella Mandragola, il testo del Machiavelli letterato in assoluto più studiato, certo perché il più divertente ed il più ricco e che, più di ogni altro, ha dato adito alle interpretazioni più diverse. L'assenza di ogni notizia certa sulle motivazioni esterne e contingenti che spinsero l'autore alla scrittura della Mandragola (le ipotesi di Ridolfi, pur affascinanti e probabili, restano sempre nel campo delle ipotesi) e soprattutto l'assenza di ogni certezza sui tempi intercorsi tra l'ideazione del testo e la materiale stesura dello stesso, non permette di supporre una ipotetica fretta, o causa simile, che possa giustificare la necessità di impiegare situazioni e lessico tipicamente decameroniani. Del resto, anche se siamo cronologicamente in tempi sicuramente anteriori all'imposizione bembesca del dettato boccacciano, non poche altre sue opere attestano come Machiavelli fosse "penetrato" della conoscenza del Decameron al punto che le situazioni e le citazioni prese da Boccaccio sono diventate parte pregnante e totalmente integrata della sua scrittura, della sua memoria letteraria.

L'identificazione di Nicia con un Calandrino con la laurea è voluta dallo stesso Machiavelli che proprio nel corso del testo richiama le famose «galle confettate in uno aloè patico fresco» (Dec., VIII 6, 39) che vengono date, nella notte degli inganni, a un Messer Nicia già di suo ridicolmente camuffato, per far sì che la sua voce non venga riconosciuta (a. IV, sc. 9), con uno di quegli escamotages machiavelliani totalmente gratuiti che rendono sublime il comico della commedia: Nicia infatti, durante la spedizione notturna, ha a che fare unicamente con personaggi che conosce già benissimo e con cui ha combinato la cattura del garzonaccio che è il fine della missione; la scena del suo sputac-

2. Girolamo MuZIO, Lettere, (edizione e commento a cura di Anna Maria NEGRI), Alessandria: Edizioni dell'Orso, 2000, p. 243-244.

3. Ibid. p. 243. 
chiare l'amaro non è per nulla necessaria o funzionale al dipanarsi della storia mentre, al contrario, l'aloè dato a Calandrino era la chiave della beffa e la dimostrazione effettuata di fronte ad un pubblico numeroso ed ignaro di una colpevolezza programmata da Bruno e Buffalmacco; che poi la stupidità dell'uno non sia inferiore a quella dell'altro fa parte dell'elegante gioco di richiami effettuato nella commedia. Ugualmente non è difficile l'accostamento, se non l'identificazione, di Nicia con mastro Simone, che aveva "studiato assai» per conquistare il diritto di porre fuori della sua porta quell'insegna che Bruno gli dipinge; Messer Nicia rappresenta, per di più, la figura del beffato che alla fine della beffa è soddisfatto e contento; per lui sarebbe inconcepibile, come invece accade per mastro Simone, una rivalsa nel corso del Decameron: il dottore che è personaggio attivo nella novella di Calandrino incinto (Dec., IX 3), dimostra, all'interno dell'universo decameroniano, l'eterna possibilità di trovare uno sciocco che sia più sciocco anche di colui che pare aver raggiunto il massimo livello di credula stupidità (cfr., a proposito, la descrizione di Mastro Simone in Dec., VIII 9). Ugualmente le novelle di Decameron VII 7 (Ludovico e madonna Beatrice) e II 9 (Bernabò e Ginevra) sono state ricordate come ben presenti nella commedia. ${ }^{4}$

In questa sede non si evidenzierà ulteriormente la derivazione boccacciana della Mandragola, ma la Mandragola sarà orientata in funzione della Clizia. Ben certi, sicuramente, che il buon Machiavelli, nella sua scrittura di questa commedia, si era "esercitato non poco»: ovviamente la definizione non va certo letta nell'accezione letterale totalmente errata, espressa dal Mazzoni, ${ }^{5}$ ma in altro significato: nel trascorrere degli anni che passano dalla Mandragola alla Clizia sembra si possa trovare un risvegliato interesse per lo studio della macchina teatrale degli antichi, che, nell'ambito anche degli Orti Oricellari si veniva nel tempo disvelando, e alle cui discussioni il nostro pare essere stato testimone. Nella scrittura della Clizia, infatti, lo stesso Machiavelli sembra allontanarsi e sfuggire dalle situazioni ironicamente boccaccesche che egli stesso aveva poco tempo prima creato per rifugiarsi in un linguaggio estremamente più piano e posato: in questa direzione la rievocazione e l'immediato abbandono del personaggio di fra' Timoteo appena fatto risorgere dalla Mandragola è dei più significativi. Ora se pur è vero che la Clizia fu scritta in tempi estremamente brevi ${ }^{6}$ e con obblighi di riconoscenza e di passione (per il Fornaciaio e la Barbera) che egli sentiva molto forti, è anche ben vero che Machiavelli non è scrittore che si ritragga di fronte al piacere della battuta o della descrizione della situazione salace ed ironica: un numero altissimo di lettere, scritte a personaggi i più diversi e ben più degni di «rispetto» di quelli che egli trovava ospiti di Jacopo Falconetti, ne sono prova.

4. Cfr. Michelangelo PICONE, «Struttura della Mandragola», Rassegna europea di letteratura italiana, n. 19, 2002, p. 106-107.

5. Niccolò MACHIAVELli, Tutte le opere storiche e letterarie, (a cura di Guido MAZZONI e Mario Casella), Firenze: G. Barbera, 1929, p. XLVI.

6. Roberto Ridolfi, Vita di Niccolò Machiavelli, Firenze: Sansoni, $1969^{4}$ [Roma: Belardetti, 1954], p. 325. 
Per quel che riguarda la Clizia la critica ha giustamente sottolineata la derivazione plautina dalla Casina, ed ha portato a riprova paragoni e collazioni non sempre persuasive ed esaustive, fortunatamente talvolta intelligenti e precise come nel caso della lettura che ne ha fatto, in tempi ormai remoti, Luigi Vanossi. L'osservazione che «la splendida invenzione plautina viene abbassata di tono, portata ad un livello quasi umile, di prosa quotidiana, generando un effetto che non ha più nulla della parodia letteraria, ma che diremmo piuttosto grottesco" ${ }^{7}$ è indubbiamente impregnata di verità. Verrebbe, per la lingua ed i «sali» della Clizia quasi da ritorcere a Machiavelli l'osservazione che lui nel Dioscorso o dialogo intorno alla nostra lingua fa nei confronti della lingua comica ariostesca ed accusarlo di usare qualcosa di cui non è totalmente padrone. ${ }^{8}$ Machiavelli sembra qui aver abbandonato la lingua e l'ispirazione boccacciana, che erano stati fondamentali e basilari per forgiare l'incredibile, geniale miscuglio della lingua creata nella Mandragola, per far diventare suo il linguaggio teatrale che stava diventando predominante, quello dell'imitazione plautina e terenziana, lingua (e linguaggio) che, come il testo della commedia dimostra, non riesce a possedere e dominare in toto. Anche se sappiamo benissimo che le esercitazioni di traduzione (dalle perdute Maschere, all'Aulularia forse da lui tradotta e poi sfruttata dal Gelli, alle due versioni dell'Andria) sono cronologicamente più vicine alla Mandragola che alla Clizia, è con quest'ultima commedia che Machiavelli sembra ricercare un avvicinamento alla lingua dei commediografi latini, rinunciando ai «sali» che solum erano suoi e creando quindi una lingua asettica, perfetta, ma che non produceva esiti di comicità; $o$ meglio comica solo per intervalla quando l'autore torna o si lascia scappare la realtà linguistica che più gli si confa. Del resto, anche per quel che riguarda la traduzione dell'Andria nelle due versioni (autografe) che sono in nostro possesso, si può dire che le uniche battute che davvero si imprimono nella mente, in cui vediamo una zampata che abbia qualche cosa di machiavelliano, sono quelle in cui egli si allontana formalmente (non certo contenutisticamente) dal dettato originario. Esempio: Quid his vult? terenziano che diventa Che vuole questo cazzo? Con variante questo zugo: e quel termine volgarmente pregnante connota perfettamente il personaggio che lo pronuncia.?

Machiavelli che traduce Terenzio ed imita Plauto è solo eccezionalmente un Machiavelli comico. Nonostante tutto questo, io sono però convinta che, pur ponendosi ad una distanza siderale dalla Mandragola, la Clizia sia generalmente stata, dalla critica, considerata e trattata peggio di quello che meritava, quasi che il suo vero merito fosse solo quello di riportare sul frontespizio il nome del Segretario fiorentino. Per convincersene credo sia sufficiente ritornare alla commedia dopo aver fatto l'esperienza della lettura di una decina,

7. Luigi VANOSSI, «Situazione e sviluppo del teatro machiavelliano», in Lingua e strutture del teatro italiano del Rinascimento, Padova: Liviana, 1970, p. 91

8. Niccolò MACHIAVELli, Discorso intorno alla nostra lingua, (a cura di Paolo Trovato), Padova: Antenore, 1982, \$69.

9. Credo sia da ricordare che il termine è usato anche da Messer Nicia: «m'hanno qui posto come un zugo a piuolo» (a. III, sc. 7). 
quindicina di altre commedie del Cinquecento (per esempio dopo quelle, pur scelte fior da fiore, presenti nella raccolta fatta da Borsellino) ${ }^{10}$ invece che, come siamo abituati, dopo la Mandragola. Fatta questa premessa non avrei dubbi nel sostenere che, a mia impressione, la parte della Clizia che teatralmente «tiene» è, in toto, boccacciana.

\section{Le donne}

Ferondo ha per moglie «la miglior donna che fosse nelle tue contrade [...] e la più dolce» (Dec., III, 8, 50-51) ed anche Nicia aveva vicino «la più dolce persona del mondo e la più facile» (Mandragola, a. III, sc. 2). Nella Clizia, invece, non ci sono donne dolci e facili: con l'esclusione, forse, di Clizia stessa, fanciulla diciassettenne che non compare in scena, di cui solo sappiamo che, da quando aveva cinque anni, era «tutta gentile» (Clizia, a. I, sc. 1): le altre donne sono costrette, per il comportamenti degli uomini che deviano dalle regole del «retto» vivere, ad abbandonare questo atteggiamento di fiducia nei loro confronti e a "stare in orecchi come la lepre» ${ }^{11}$ o doversi mantenere sempre in allerta e in guardia perché "ogniuno l'ha posto il campo intorno». ${ }^{12}$ Esse non hanno mai «voluto il giuoco» dei loro uomini ma ne sono state costrette, proprio per non finire derise ("tu sei quello che l'hai voluto [il giuoco] di tutti noi» (Clizia, a. V, sc. 3). La semplice ingenuità e la fiducia diventano elementi negativi di una personalità, quando non possono basarsi su garanzie reali.

Lucrezia e Nicia partono da una posizione matrimoniale ideale, di fiducia dell'uno verso l'altra ${ }^{13}$ e di ambedue verso il mondo; essi sono gli unici personaggi creduli della Mandragola, coloro che prestano fede alle virtù della pozione, ai giuramenti di fra' Timoteo («vi giuro per questo petto sacrato»). In questo atteggiamento fiducioso Messer Nicia permane, beatamente sprovveduto, fino alla fine della commedia, mentre Lucrezia viene «svegliata» dalle sue candide illusioni di onesto e rigoroso ordine, dalla rivelazione dell'inganno che le viene presentata apertamente da Callimaco; Lucrezia diventa allora "onorevolmente cattiva», ${ }^{14}$ senza, però, mutare la sua incrollabile fiducia in un regolamentato ordine delle cose; ${ }^{15}$ seguendo questa sua nuova «etica» sente la necessità, il bisogno di trasgredire fino in fondo. Pur essendo assolutamente consapevole che, come una "figliola di Lotto» (Mandragola, a. III, sc. 11)

10. Nino Borsellino (a cura di) Commedie del Cinquecento, 2 voll, Milano: Feltrinelli, 19621967.

11. «Da quel tempo in qua ella sta in orecchi come la lepre; e come se le dice nulla, ella vi fa dentro mille difficultà»: Mandragola, a. III, sc. 2.

12. «E” mi bisogna guardare questa fanciulla dal figliuolo, dal marito, da' famigli: ognuno le ha posto il campo intorno": Clizia, a. II, sc. 3. La Clizia è citata dall'edizione critica in Daria Perocco, "Il testo della Clizia", in Gennaro Barbarisi e Anna Maria CABrini (a cura di), Il teatro di Machiavelli, Milano: Cisalpino, 2005, p. 438-487.

13. Ne è prova il fatto che Lucrezia si affretta a raccontare a Nicia perché non vuole più «tornare a' Servi».

14. Luigi Russo, Machiavelli, Bari: Laterza, 1966 [1945], p. 108.

15. Cfr. Mandragola, a. V, sc. 4. 
non aveva commesso peccato nella prima "giunta», dato che la sua volontà non era implicata nel peccato, si rende totalmente colpevole da quando accetta Callimaco come amante stabile e renderà il peccato ancora più grave perché consumerà l'adulterio con qualcuno che lei stessa ha contribuito a far diventare suo parente ("faratti nostro compare»); sia lei che Nicia sono ancora convinti della sacralità e della strettezza del nodo di parentela che il comparatico ha in sé. Dionisotti ha giustamente sottolineato l'importanza del comparatico in ben due novelle del Decameron: ${ }^{16}$ frate Rinaldo, con una retorica degna di fra' Timoteo ma in fondo ben più sana per il fine cui è applicata, trova necessario persuadere la comare con un bellissimo sillogismo. Anche Lucrezia (dimostrando ancora una volta quel lato del suo carattere che vuole che lei ricerchi il massimo, la perfezione in qualunque atteggiamento della sua vita) vorrà peccare fino in fondo compiendo quindi una specie di incesto con colui che proprio lei ha eletto non al ruolo unicamente di amante, ma di parente, facendolo diventare suo compare. In Nicia la proposta del comparatico può solo procurare una ulteriore rassicurazione di cui si può essere certi, «il dottore» non aveva bisogno, data la sua assoluta fiducia nella moglie e nel falso medico; egli è sicuro che Callimaco sarà solo l'amico, anzi lo stretto parente con cui mai lei potrà avere nessun genere di contatto che evada dal lecito, colui che procurerà loro «un bastone per la vecchiaia» certo non ipotizzando il derisorio ritorno della metafora che la frase comporta. Del resto la risposta di Nicia alla aperta proposta di rendere Callimaco loro compare è un: «Oh, benedetta sia tu!» che ripete puntualmente, con un bel chiasmo fra il maschio e la femmina, fra colui che sa e colui che ignora, il «benedetta sie tu» di Rustico ad Alibech (Dec., III 10 20). Esclamazione questa, del resto, che nel Decameron compare sempre in contesti comici e di beffa (oltre ad Alibech, Ser Ciappelletto I 1, 40 e 52; Angelo Gabriello IV 2, 31; Frate Puccio III 4, 15; Frate Cipolla VI 10, 51; Calandrino innamorato IX 5, 47 e Calandrino e il porco imbolato VIII 6, 35). Nell'ultima scena della Mandragola nessuno degli altri personaggi, che paiono ben conoscere la ripresa boccacciana del tema (in Dec., VII 10), si preoccupa minimamente delle possibili conseguenze, sicuri, come Tingoccio riferisce a Meuccio che si informa a proposito, che nell'aldilà «non si tiene ragione alcuna delle comari» (Dec., VII 10, 28).

Lucrezia è dunque una Catella che non solo si adatta di buon grado alla situazione in cui forzatamente e contro la sua disposizione («mai per me medesima arei fatto" Mandragola, a. V, sc. 4) viene posta, ma la esaspera e, seguendo la sua natura, la porta fino in fondo.

Per quel che riguarda le considerazioni dell'autore sulle donne bisogna ben dire che in Machiavelli non si ritrova certo quella positiva attenzione che avevamo trovato nel Boccaccio del Decameron o la denigratoria (e impaurita) ammirazione del Corbaccio. Quando Callimaco, parlando a se stesso, cerca di trovare una qualunque soluzione alla sua angoscia d'amore, si esorta: «non ti prosternare, non t'invilire come una donna» (Mandragola, a IV, sc. 1). Anche Lucre- 
zia che pure è «savia, costumata e atta a governare un regno" secondo Ligurio, (a. I, sc. 3), «savia e buona» per fra' Timoteo (a. III, sc. 9), come tutte le donne «ha poco cervello» (a. III, sc. 9). Sostrata «è bene una bestia»: sempre per fra' Timoteo «tutte le donne hanno poco cervello» e se pure Lucrezia dimostra una certa superiorità sulle altre è solo perché «in terra di ciechi chi v'ha un occhio è signore». Sofronia è accusata di essere «un poco roza e non ti sai accomodare con le persone» (Clizia, a. IV, sc. 5). Sostrata poi, oltre ad essere stata «buona compagna" nel passato, ora "è della opinione nostra» e cioè disposta a non farsi troppe domande, come lo stupido Nicia.

Il fatto che le donne risultino sempre vincitrici nelle battaglie che egli stesso rappresenta e mette in scena non sembra far cambiare l'opinione diminutiva che Machiavelli dimostra di avere (o, almeno, sicuramente fa manifestare ai suoi personaggi).

\section{I vecchi e i giovani}

I vecchi dovrebbero essere saggi, ed aiutare i giovani con la loro esperienza: nulla in verità di meno reale nel teatro machiavelliano. Se Nicomaco, non ancora travolto dalla frenesia amorosa «ragionava con el figliuolo, amonivalo, davali a conosciere gli uomini e con qualche exempio antico e moderno gli insegnava vivere» (Clizia, a. II, sc. 4), il precipitare nella passione, in fondo nella vita vera e non preordinata, gli fa totalmente perdere il senso del limite dentro il quale sembrava voler restare: ma questo non toglie che in lui continui a rimanere ferma la convinzione che egli è comunque nel giusto, è il padrone e vuole rimanere «signore di casa mia» (Clizia, a. III, sc. 1). Il problema del vecchio Nicomaco è non avere accanto un Ligurio che lo consigli. Se si può pensare un parallelo tra le due commedie machiavelliane ed accostare le coppie amante e consigliere Callimaco- Ligurio / Nicomaco -Damone si vede subito che nel primo caso ci si trova davanti all'incertezza del giovane Callimaco, che non sa cosa fare, dubita e chiede consiglio, vuole aiuto sia sull'organizzazione dell'inganno che sul modo da tenere per tornare con la donna anche dopo averla avuta, pur avendo a disposizione tutte le possibili carte da giocare: è ricco, giovane, colto ecc. L'esito felice dell'inganno amoroso è affidato a Ligurio, altrettanto giovane, con il «sangue [che] si confa» con quello di Callimaco, ammiratore di Lucrezia, ma certo non innamorato, e che fattivamente sarà colui che contribuirà a porlo al buio e con un inganno nel letto di lei: come Gisippo, che stimava l'amicizia molto più dell'attrazione per una donna, aveva fatto con Tito e Sofronia (Dec., X 8); già in Boccaccio Gisippo, nella coppia di amici, era il meno ricco, il meno preso dalla donna e colui che "giocava in casa" (la prima parte della novella, che qui interessa, si svolge ad Atene ed ateniese è Gisippo mentre romano è Tito): stessa situazione di Ligurio, che non è stato favorito dalla fortuna e agisce in una Firenze da cui sembra non essersi mai allontanato, contrariamente al "parigino" Callimaco.

Nicomaco è invece sicuro di se stesso e dell'esito felice dell'inganno preparato; è pronto a giocare un altro inganno se l'esito del sorteggio (da lui pro- 
posto) gli fosse negativo (Clizia, a. III, sc. 6: «e se pur la sorte mi venisse contro, io ho pensato al remedio»): addirittura un'altra beffa, un altro raggiro di cui non sappiamo nulla; forte di queste sue certezze, rassicura anche l'amico Damone (Clizia, a. IV, sc. 11). La sicurezza dei vecchi (anche quando sbagliano) è presente in molti casi boccacciani, là dove essi perseguono le loro decisioni, dichiarano di sapere quello che fanno e ne vanno orgogliosi, pur se hanno commesso errori. Si veda solo l'esempio di Riccardo di Chinzica (una novella che è stata dai critici più volte richiamata come ispiratrice della Mandragola) che non riesce a capire che è stata una aberrazione imputabile solo a lui il volere una moglie giovane e per non ammetterlo a se stesso finisce nella follia. Lo sbaglio di Nicomaco nel gestire la trama dell'inganno si nasconde nell'eccessiva sicurezza che lo contraddistingue, nella certezza del favore della Fortuna che lo ha assistito; il peccato di conseguenza è consistito nell'abbassare la guardia, lasciandosi già andare con l'immaginazione alla realizzazione del sogno proibito, e sottovalutare le reazioni che l'orgoglio ferito di una moglie e le potenzialità dell'inganno femminile potevano creare.

Giustamente ha osservato Giorgio Inglese che l'«ordine» di Nicomaco è mortalmente noioso, anzi "grigio, conformistico, opprimente-prima; anche più grigio e più opprimente dopo l'oscena umiliazione del protagonista». ${ }^{17} \mathrm{Il}$ discorso è fatto dal critico tenendo soprattutto conto degli enunciati presenti nel Prologo della commedia. Ma, guardando al personaggio e a tutte le sue battute, bisogna notare che è Nicomaco stesso che del mantenimento di questo grigio ordine si preoccupa, augurandosi che tutto rimanga uguale e nulla nelle abitudini quotidiane sia mutato: si veda ad esempio, nell'atto II sc. 2, quando nel dialogo con Pirro, suo complice, è proprio Nicomaco ad essere particolarmente preoccupato di "far le cose in modo che la casa non vadda sottosopra». Il vecchio protagonista della Clizia vuole la diversione da ciò che è abitualmente praticato, la rivoluzione (con l'entrata della ventata sessuale) solo per se stesso, senza che nessuno degli altri personaggi tragga dei veri, considerevoli vantaggi dalla sua deviazione dal seminato: non è difficile comprendere che è per questo motivo che la beffa non può avere un esito positivo ed una riuscita conforme ai suoi desiderata. Nel Boccaccio, in numerose novelle (una per tutte, quella di frate Puccio, Dec., III 4) non solo il beffatore, ma anche il beffato è convinto di avere ottenuto un notevole guadagno; nella Mandragola tutti (davvero tutti) i personaggi escono dalla notte degli inganni con un tornaconto (Nicia col «naccherino», Sostrata con la sicurezza del futuro suo e della figlia prima accanto e poi come sicura erede di un genero ricco, Lucrezia con un amante ed «un bel figliolo maschio», Timoteo con «i denari della limosina» ecc.). Nella Clizia Sofronia che pure sembra rassegnata al fatto di essere ormai giunta a quello stadio della sua vita in cui non ci possano per lei essere soddisfazioni diverse e più concrete di quell'ordine regolato, quella messa, quelle amicizie

17. Giorgio InGLeSE, “"Le stesse cose ritornano”. Considerazioni sulla Clizia», in Gennaro BARBARISI e Anna Maria CABRINI (a cura di), Il teatro di Machiavelli, Milano: Cisalpino, 2005, p. 492, ora in ID., Per Machiavelli, cit., p. 186. 
femminili con donne della sua età, che costituiscono la struttura della sua vita, non tollera che il marito voglia «il giuoco» di lei e di «tutti noi altri», la gratuita rivoluzione di quell'universo casalingo di cui si sente, a ragione, padrona. Anche se non è stata "buona compagna" (ma, se posso ipotizzare un paragone, io la vedo più vicina ad una Sostrata che ad una Lucrezia invecchiata), sa però come vanno le cose al mondo: è infatti sua la più ironica e divertente battuta della commedia: "Gran miraculo un frate fare ingravidare una donna! Miraculo sarebbe se una monaca la faciesse ingravidare ella». ${ }^{18} \mathrm{E}$ certo anche per lei l'allegria, il sano desiderio di rivalsa scappa fuori («questa tua donna sarà come le mezzine da Santa Maria Impruneta»), ${ }^{19}$ divide con l'amica e coetanea Sostrata (il personaggio della Clizia, moglie di Damone, non quella omonima, ben più spericolata, della Mandragola) il piacere per la battuta ironica, magari leggermente equivoca nei confronti dei mariti, che ancora le fa ammiccare.

Contrariamente a Nicia, che non sa (ne è totalmente incapace) e quindi non vuole creare una situazione in cui agire, e delega agli altri sia l'idea che l'azione, Nicomaco cerca continuamente di creare azioni che gli permettano di arrivare al suo fine: spinto dall'impossibilità dell'inazione, di non realizzare il suo desiderio amoroso, sembra quasi incarnare la necessità presente nel boccacciano (che diventerà poi machiavelliano): «è egli meglio fare e pentere che starsi e pentersi» (Dec., III 5, 30). Nicomaco, come Nicia, compie anche un altro errore: nella sua ostinata sicurezza vede solo quello che vuole vedere; per l'uno e per l'altro dei due vecchi le persone travestite incarnano davvero quelle che i due protagonisti immaginano: anche di fronte all'evidenza di caratteri somatici che non corrispondono, da una parte Nicia non ha dubbi su fra' Timoteo vestito da Callimaco: «voi contraffate bene la voce»; ${ }^{20}$ dall'altra Nicomaco è pronto a giustificare l'altezza della falsa Clizia che è impersonata da Siro travestito: «Hai tu veduto come l' è grande? La si debbe essere aiutata con le pianelle». ${ }^{21}$ Così tutti gli sciocchi del Decameron non avevano riconosciuto i loro beffatori travestiti.

Sempre nella dinamica machiavelliana della contrapposizione tra vecchi e giovani è da notare che i travestimenti dei giovani vanno a buon fine (v. Callimaco/garzonaccio nella Mandragola e Siro/Casina nella Clizia), quelli dei vecchi invece non riescono (Nicomaco/Pirro: e non sappiamo neppure se la vecchiaia poteva essere stata riconosciuta al buio!). Lo scambio di persona è elemento fondante nel teatro latino, ma è certo anche base della novellistica di beffa; il caso di Dec., III 6 (Ricciardo Minutolo e Catella), il più famoso e quello sempre evocato come ispiratore della beffa che sta a base della Mandragola, non è certo l'unico cui il nostro si poteva rifare.

Altra grande paura è quella che «la cosa si risappi». "Purchè la cosa non si risappia» ripete Nicomaco più volte e, per quel che riguarda il vecchio protagonista della Clizia, il dubbio che sorge è chi possa essere l'eventuale destina- 
tario del pettegolezzo. Nel suo giro familiare, ampliato come tutti quelli del tempo, tutti sanno e tutti ridono ("e così ogniun rida e Nicomaco pianga») ed anche in a. I, sc. I Cleandro dice «li è una tresca il fatto nostro». Diversamente da mastro Simone, che dai racconti fatti da Bruno e Buffalmacco avrebbe potuto avere notevole detrimento nella sua clientela, Nicomaco può temere solo un generico ambiente borghese, fatto da tanti Ramondi napoletani che arrivano per caso. Molto più significativa e pregnante era stata la preoccupazione per il silenzio sull'azione compiuta di fra' Timoteo che basa, però, la sua sicurezza nel coinvolgimento di molte persone ("quando una cosa importa a molti, molti ne hanno a avere cura») o quella di messer Nicia («ma, sopra a tutto che non si sappia, per amore degli Otto!»: Mandragola, a. II, sc. 6).

Qualche critico ha voluto accennare ad un "erotismo" presente nelle commedie machiavelliane. Bisogna dire che Machiavelli è forse più di Boccaccio ironico e denigratore per quel che riguarda le abilità sessuali dei suoi personaggi. Se è vero, come ha detto Ridolfi, che Niccolò si rifletteva nelle prime lettere dei suoi due protagonisti vecchi (NICia e NIComaco) bisogna per di più anche notare che non solo il nome, ma anche l'età di Machiavelli coincide con quella di un suo protagonista: messó alla berlina: quando, infatti, scrisse la Mandragola, egli aveva circa 49 anni: «se non è giovane, non è al tutto vecchio come pare»: afferma Callimaco di Nicia; la frase è uguale a quella che un altro beffato stupido e famoso, Calandrino, afferma di se stesso, quando si innamora: "che io non son vecchio come io ti paio» (Dec., IX 5, 36). Tutto questo, ovviamente, in ironica coincidenza con l'innamoramento per la Barbera che gli dava "più da pensare che lo Imperadore». ${ }^{22}$ Nelle commedie machiavelliane i giudizi sulla validità sessuale, positiva o negativa, sono sempre dati da uomini (l'autodefinizione di «rubizzo e ferrigno» è di Nicia) mentre la «differenzia dalla iacitura mia a quella di Nicia», come è già stato notato, è vanto di Callimaco, non è parte del serissimo (e per niente erotico) discorso di Lucrezia. Nella Clizia assistiamo ad una serie di dimostrazioni di "procurata potenza" quando Nicomaco elenca tutto quello che sta per ingerire per arrivare in forma all'incontro amoroso. Nel Decameron, invece, tutti sappiamo che le più feroci critiche vengono proprio dalle donne (Monna Tessa, Bartolomea de Gualandi, Catella, ecc.). Sottolineata come testimonianza di una presunta omofilia di Nicia, la Mandragola riporta la testimonianza della gagliardia di colui che viene cacciato nel letto di Lucrezia ("dell'altre cose non me ne domandate» a. V, sc. 2); nella Clizia non c'è la "prova" della validità delle carni che staranno con Clizia, come invece aveva fatto Nicia con Callimaco. Il controllo è proposto dal giovane Pirro («dubito che la vecchiaia non si riconosca al buio» [Clizia, a. IV, sc. 2]) ironicamente disposto ad aspettare il suo turno, ma allontanato dal vecchio Nicomaco, che ha la sicurezza di «rompere una lancia con Clizia» perché si «sente gagliardo come una spada» (Clizia, a. IV, sc. 11). I suoi tremori (dovuti all'età) «Che [...] ho io [...] intorno agli occhi? E' mi pare 
havere e bagliori, che non mi lasciono vedere lume» ${ }^{23}$ assomigliano a quelli del giovane Callimaco che notava: «le gambe tremano, le viscere si commuovono... gli occhi abbarbagliono"; ${ }^{24}$ non credo sia casuale che ambedue i personaggi recitino le loro ansie di innamorati, posti da soli sulla scena e ad inizio di atto: non è certo qui il caso di ricordare l'importanza che il monologo riveste nella resa teatrale. L'uomo "abbarbagliato" non è in grado di condurre un inganno: anche Calandrino desidera, nelle sue smanie d'amore "chi lo aiutasse» (Dec., IX 5, 13). Al di là dell'amore, la vecchiaia si presenta come un problema, per chi non ne sa trarre le giuste gioie, che non sono più quelle della giovinezza. Boccaccio sembra essere molto più comprensivo: la novella $\mathrm{I} 10 \mathrm{e}$ l'introduzione alla quarta giornata ne sono valida prova; è una donna (vecchia) che in V 10, 18 in un paragone fra uomini e donne, attesta che «degli uomini non avvien così: essi nascono buoni a mille cose, non pur a questa, e la maggior parte sono da molto più vecchi che giovani; ma le femine a niuna altra cosa che a fare questo e figliuoli ci nascono». Nessuna speranza invece esce dal dettato di Machiavelli, a proposito deciso ed assoluto nelle sue affermazioni, come attesta la Canzona che segue il secondo atto della Clizia:

Quanto in cor giovenile è bello amore

Tanto si disconviene

In chi delli anni suoi passato ha il fiore.

Amore ha sua virtute agli anni uguale

E nelle fresche hetati assai sionora

E nelle antiche poco o nulla vale:

Sì che, o vecchi amorosi, el meglio fora

Lassar l'impresa a giovanetti ardenti,

Ch'a più forte opera intenti,

Far ponno al suo signor più largo honore.

La canzone della Clizia, come quelle della Mandragola riveste una notevole importanza per l'interpretazione della poetica dell'autore ${ }^{25}$ Nicia, Nicomaco e Riccardo di Chinzica che hanno "scoperto la croce della carnalità come un destino inevitabile per ogni stagione della vita dell'uomo», ${ }^{26}$ hanno anche in comune la totale mancanza di percezione dello scorrere del tempo, che per loro, nelle cose d'amore, è definitivamente tramontato nei ritmi e nei modi della giovinezza.

\section{Il gioco delle parole}

Il ritmo vivace e sostenuto della commedia deve lasciare attimi di sospensione per catturare l'attenzione dello spettatore, a cui non si può e non si deve spie-

23. Clizia, a. II, sc. 1.

24. Mandragola, a. IV sc. 1.

25. Arnaldo BRUNI, "Gli intermedi della Mandragola», in Gennaro BARBARISI e Anna Maria CABRINI (a cura di), Il teatro di Machiavelli, p. 367-408.

26. Ibid., p. 392. 
gare la trama se non con rischio e pericolo di noia. Così anche nelle novelle comiche, in particolare quelle di beffa o di motto, dove l'attesa per la soluzione dell'intrigo imprevisto è chiave per catturare l'attenzione. Non meraviglia dunque trovare riprese dal Decameron che danno nella commedia i tempi di sospensione e di attesa: L' "andrai seguitando il mio parlare e accomodantoti a quello» di Ligurio (Mandragola, a. I sc. 3) è espediente (e frase) che si ritrova più volte nel Boccaccio messa in bocca a personaggi, soprattutto femminili che il destino ha posto a rischio, che non hanno tempo di spiegare, che si fidano della intelligenza del partner e prendono in mano la regia della commedia che sta per essere messa in scena, dove il deuteragonista è totalmente dipendente dalle mosse del primo attore: e credo non sia azzardato parlare di spazio scenico e di quinte teatrali nella novella terza della settima giornata quando la comare raccomanda a frate Rinaldo «ascolterete bene ciò che io gli dirò, sì che le vostre parole poi si accordino colle mie», ${ }^{27}$ o nella novella sesta sempre della settima giornata dove gli attori che si muovono secondo la regia femminile, senza conoscere il copione, sono addirittura due. Nella giornata V 10, 28 la moglie di Pietro da Vinciolo nasconde il marito mancato (il giovane che si è fatta venire in casa) sotto una cesta da polli, mentre, nella Clizia, Pirro, marito mancato perché ha ceduto lo ius primae noctis a Nicomaco "si è fuggito in cucina, e si è nascosto drieto a la cesta de capponi» (Clizia, a. IV, sc. 7): non si può non pensare al parallelismo dei due giovani che sono impediti a compiere l'atto sessuale da elementi esterni, mentre i due vecchi sono bloccati da elementi fisici (l'omosessualità di Pietro e la pochezza di Nicomaco). L'asino che compare nella novella di Boccaccio, e che, con chiara allusione apuleiana, nasconde e rivela, trova come parallelo il «pugnale» afferrato da Clizia, che verrà trovato da Nicomaco nel letto quando tenterà di «rompere una lancia» con lei. A differenza della Clizia, però, dove la vecchiaia e la burla fanno sì che nella notte accadano solo «rovigamenti di vestirsi, di aprir usci, di scendere e di salire in sul letto", ${ }^{28}$ tanto rumore e niente fatti, nella novella di Boccaccio, dove all'autore è «uscito di mente» che cosa sia accaduto, i fatti abbondano tanto che il giovane, la mattina seguente, è incerto «qual più stato si fosse la notte o moglie o marito» (Dec., V 10, 63).

Alcuni termini delle commedie machiavelliane denunciano una ripresa diretta dal Decameron, altri denotano solo una presenza che fa notare la coincidenza, pur non dando sicurezza di derivazione. Un esempio può essere dato da vituperio e vituperare che sono termini di Lucrezia nella Mandragola, di Nicomaco nella Clizia, ed in Boccaccio compaiono nella novella di Barnabò e Ginevra "da questo traditor d'Ambrogiol falsamente e reamente vituperata» (II 9, 68); Ricciardo e Catella «vituperar voi e mettere in pericolo e in briga vostro marito e me» (III 6, 45); poi in Pietro da Vinciolo (V 10, 44: «universal vergogna e vitupero di tutte le donne [...] non s'è vergognata di vituperare»); "minacciando di vituperarla se non facesse il piacer suo» (VII 6, 6 
Isabella, Leonetto e Lambertuccio); "per paura che questo suo vitupero non palesassero" (VIII 9, 112 Mastro Simone Bruno e Buffalmacco). Si può notare che, se è pur vero che vituperare vituperevole, vitupero vituperosamente e derivati ricorrono nel Decameron con una certa frequenza, il termine si ripresenta e ricorre nelle novelle che sono state indicate come quelle che maggior spunto contenutistico hanno dato a Machiavelli.

Una ripresa diretta si può poi notare dalla nov. III 8, 25: «la santità dimora nell'anima e quello che io vi domando è peccato del corpo", che non può non richiamare il discorso di fra' Timoteo a Lucrezia; ${ }^{29}$ nella stessa novella, al pargrafo 31, compare «in un bicchier di vino non ben chiaro ancora... gliela [polvere] diè a bere»: è stato notato che quel vino ha molto in comune col bicchiere di hyppocras che sostituisce la pozione alla mandragola per Lucrezia (anche se l'effetto soporifero della durata di tre giorni che la polvere sciolta in quel vino avrà, rievoca piuttosto una Giulietta ben di là da venire).

Le descrizioni più derisorie, oltre che dal Decameron dichiarano una ripresa diretta dal Corbaccio; si veda, come esempio, il brano della Clizia in cui il giovane Cleandro immagina, tra le braccia del vecchio padre, il corpo dell'amata e di conseguenza accadrà che

...sì dilicato viso sia da sì fetida bocca scombavato e sì delicate carni da sì tremanti mani e da sì grinze e puzzolente membra toche ${ }^{30}$

Come nel Corbaccio l'autore rievocava non senza orrore la disposizione femminile di darsi ad ogni uomo purché ricco, senza alcun problema

che esso sia vecchio bavoso, a cui colino gli occhi e triemino le mani e il capo [...] le membra i capelli e il viso..tanta morbidezza sottomettere, porgere e lasciare trattare alle mani paraletiche alla bocca sdentata bavosa e fetida. ${ }^{31}$

Nelle riprese machiavelliane del Boccaccio comico c'è un divertimento dell'autore nel compiere un gioco equivoco con i termini usati: l'equivoco può sembrare della stessa natura, ma in un autore può addirittura diventare parodia del significato di quello che è nell'altro: Machiavelli non si fa mai «giugnere», né «sulla bontà», come Lucrezia, né «in sul furto», ${ }^{32}$ come Nicomaco: non per nulla la popolare espressione non compare mai in Boccaccio e sembra essere (e nei due contesti citati indubbiamente è) solum sua, ${ }^{33}$ del Segretario fiorentino: un altro modo per "fare el suo tristo tempo più suave». 34

29. In particolare il brano: "Quanto all'atto, che sia peccato, questa è una favola, perché la volontà è quella che pecca, non el corpo" Mandragola, a. III, sc. 11.

30. Clizia, a. IV, sc. 1.

31. Giovanni Boccaccio, Corbaccio, in ID. Tutte le opere (a cura di Vittore BranCA), vol. V, Milano: Mondadori, 1994, \$161-163, p. 469-470.

32. Mandragola, a. III, sc. 9.

33. La chiara allusione è alla lettera di Machiavelli a Francesco Vettori del 10 dicembre 1513: cfr. NiCCOLÒ MACHIAVELLI, Lettere, cit., p. 423-428.

34. Mandragola, Prologo, v. 50. 